\title{
Correlação empírica para viscosidade cinemática de biodiesel de fontes diversas e suas misturas com o diesel
}

\section{Paulo Gabriel Ferreira de Azevedo', Ivan Costa da Silva ${ }^{2}$, Cláudia Teresa Teles Farias ${ }^{2}$ e Luiz Antonio Pimentel Cavalcanti $^{2}$}

${ }^{1}$ Instituto Federal de Educação, Ciência e Tecnologia da Bahia. Curso de Engenharia Química. Campus Salvador. Rua Emídio dos Santos, S/№. Bairro do Barbalho. Salvador-BA, Brasil (CEP 40301-015).*E-mail: paulo97745@gmail.com.

${ }^{2}$ Instituto Federal de Educação. Ciência e Tecnologia da Bahia. Campus Salvador. Rua Emídio dos Santos, S/№. Bairro de Barbalho. Salvador-BA, Brasil (CEP 40301-015).

Resumo. 0 biodiesel é um combustível oriundo de uma matriz energética renovável e destaca-se como substituto do diesel petrolífero, já que o uso exagerado do último têm gerado consequências ambientais graves. Para o desempenho adequado do motor, o conhecimento das propriedades fluidodinâmicas do combustível é importante, o que torna viável o uso de modelos matemáticos para predição dessas propriedades. Portanto, o presente trabalho teve por objetivo propor e analisar estatisticamente um modelo para predição da viscosidade cinemática dos biodiesel de algodão (B100-A), soja (B100-S) e sebo bovino (B100-SB), e suas misturas BX nas proporções de $5 \%, 10 \%, 20 \%, 30 \%, 40 \%$ e $50 \%$ de biodiesel, na faixa de temperatura entre $293,15 \mathrm{~K}$ e $363,15 \mathrm{~K}$, com intervalos de $10 \mathrm{~K}$. 0 modelo foi proposto através da análise dos dados experimentais e os parâmetros do modelo foram obtidos através da aplicação da regressão não linear desses dados, através do aplicativo Curve Fitting, do Matlab. Foram gerados parâmetros distintos para cada tipo de biodiesel, com coeficiente de determinação mínimo de 0,993 . Superfícies modeladas que relacionam a viscosidade cinemática em função da temperatura e fração de biodiesel foram plotadas e estudadas. 0 erro máximo encontrado foi de 1,2338\% e se mostrou aceitável frente a resultados de trabalhos semelhantes. As análises de resíduos indicaram que o modelo não se mostrou tendencioso em nenhum caso estudado. 0 teste de aderência de modelos assegurou a validade estatística do modelo nos três conjuntos de dados avaliados no trabalho. Esses resultados indicam que o modelo proposto mostrou-se satisfatoriamente capaz de predizer os valores da viscosidade cinemática dos biodiesel estudados, no intervalo de temperatura analisado.

Recebido

$18 / 06 / 2020$

Aceito

$28 / 07 / 2020$

Disponível on line $31 / 07 / 2020$

Publicado

$31 / 08 / 2020$

Acesso aberto

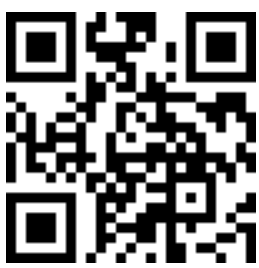

ORCID

(ㄱ) 0000-0003-0685-2928 Paulo Gabriel Ferreira de Azevedo

D 0000-0001-5990-5179 Ivan Costa da Silva

ISSN 2359-1412/RBGAS-2020-0094/2020/7/16/18/717

Rev. Bras. Gest. Amb. Sustent.

http://revista.ecogestaobrasil.net 
Palavras-chave: Biodiesel; Modelos preditivos; Viscosidade.

(D) 0000-0002-3976-613X Cláudia Teresa Teles Farias

Abstract. Empirical correlation for kinematic viscosity of biodiesel from different sources and their mixtures with diesel. Biodiesel is fuel from a renewable energy source and stands out as a substitute for petroleum diesel. For suitable engine performance, it is important to know the fluid dynamic properties of fuel, which makes it viable to use mathematical models that predict these properties. Therefore, this work aims to propose and statistically analyze a model for predicting the kinematic viscosity of cotton (B100-A), soybean (B100-S) and beef tallow (B100-SB) biodiesel and their blends, in the proportion of $5 \%$, $10 \%, 20 \%, 30 \%, 40 \%$ and $50 \%$ biodiesel, in the temperature range of $293.15 \mathrm{~K}$ to $363.15 \mathrm{~K}$, in $10 \mathrm{~K}$ intervals. The model was proposed through the analysis of experimental data and the model parameters were obtained through the application of nonlinear regression of this data, using the Curve Fitting application, from Matlab. Differents parameters were generated for each type of biodiesel, with a minimum determination coefficient of 0.993 . Modeled surfaces that relate the kinematic viscosity as a function of temperature and biodiesel fraction were plotted and studied. The maximum error observed was $1.223 \%$ and proved to be acceptable in view of the results of similar studies. Residual analyzes indicated that the model showed no tendency in any of the cases studied. The adherence test of the models ensured the statistical validation of the model in the three cases studied. These results indicate that the proposed model proved to be satisfactorily capable of predicting the kinematic viscosity values of the studied biodiesel, in the temperature range analyzed.

Keywords: Biodiesel; Predictive models; Viscosity.
(1) 0000-0003-4932-9387

Luiz Antonio Pimentel Cavalcanti

\section{Introdução}

O biodiesel é um combustível renovável que tem se mostrado uma alternativa promissora na substituição do combustível fóssil. A utilização demasiada dos combustíveis petrolíferos gera, principalmente, problemas na esfera ambiental, como poluição atmosférica e aquecimento global. Baixa toxicidade, baixa emissão de poluentes na combustão, biodegradabilidade e ausência de compostos sulfurados e aromáticos são algumas das vantagens do biodiesel (Aghel et al., 2019; Wang et al., 2020).

Constituído de ésteres alquílicos de ácido graxos, saturados e insaturados, o biodiesel é comumente empregado na forma de misturas biodiesel-diesel (mistura BX). 0 estudo do comportamento reológico desse combustível indica que sua fluidez é dificultada em baixas temperaturas, comprometendo, com isso, sua atuação no motor (Cavalcanti, 2013). Portanto, a análise das propriedades fluidodinâmicas do biodiesel é importante para assegurar o funcionamento adequado do motor, garantindo uma performance adequada por parte do mesmo, além de um processo de combustão completo, diminuindo a emissão de gases poluentes (Ramirez-Verduzco, 2013).

A etapa de atomização do combustível que opera no motor ciclo diesel está relacionada com a quantidade de energia necessária para o início da combustão, logo esse 
estágio é fundamental para a operação apropriada do motor (Cavalcanti, 2013). Segundo Wang et al. (2020), a alta viscosidade cinemática do biodiesel gera o pobre efeito de atomização, tendo como consequências, a formação de gotículas do combustível de maiores diâmetros na câmara de combustão ocasionando assim a combustão incompleta, que segundo Kanaveli et al. (2017), leva a formação grave de depósitos no motor, degradação do bico injetor e aderência do anel do pistão dificultando sua movimentação e contribui para o aumento de emissões de gases poluentes na atmosfera.

Com base nisso, entende-se a importância do conhecimento da viscosidade cinemática do BX em diferentes condições de processo. Diante da impossibilidade física e econômica da medição constante dessa propriedade, o uso de modelos matemáticos que predizem a viscosidade cinemática do BX, torna-se viável (Cavalcanti, 2016). Segundo Pham et al. (2018), essa modelagem, além de fatores econômicos, é importante na aplicação em simulação de processos, modelagem da combustão e predição dos gases de exaustão da combustão.

Desta forma, o presente artigo objetivou analisar um modelo preditivo para viscosidade cinemática dos biodiesel de algodão (B100-A), de soja (B100-S) e de sebo bovino (B100-SB) e suas misturas BX com o diesel, através de regressão não linear dos dados empíricos dessa propriedade, oriundos da literatura (Cavalcanti, 2013).

\section{Fundamentação teórica}

Com o intuito de desenvolver modelos para prever a viscosidade cinemática dos biodiesel (B100), muitos fatores devem ser levados em consideração. 0 parâmetro mais avaliado é a temperatura e como a mesma influencia na variação da viscosidade cinemática, nesse aspecto, um dos modelos mais estudados é a Equação de Andrade (Equação 1) e suas modificações (Cavalcanti, 2013; Geacai et al., 2015).

$$
v(T)=a_{1} \cdot \exp \left(a^{a_{2}} / T\right)
$$

Sendo $v$ a viscosidade cinemática em $\mathrm{m}^{2} . \mathrm{s}^{-1}$, $\mathrm{T}$ a temperatura em $\mathrm{K} \mathrm{e} \mathrm{a}_{\mathrm{i}}$ os parâmetros do modelo. Analisando a Equação 1, nota-se que a Equação de Andrade adota uma relação exponencial entre viscosidade cinemática e a temperatura, isto é, uma relação não linear. Além disso, essa equação evidencia a inversa proporcionalidade entre a viscosidade cinemática e a temperatura.

Geacai et al. (2015) avaliaram a capacidade preditiva da Equação de Andrade. Os autores estudaram dados experimentais de viscosidade cinemática das misturas binárias diesel-biodiesel, biodiesel-benzeno e biodiesel-tolueno, sendo o biodiesel produzido pelo óleo de canola, nas proporções de B10, B25, B50 e B75, na faixa de temperatura entre 293,15 K e 323,15 K, com intervalos de $5 \mathrm{~K}$. Os máximos desvios percentuais encontrados pelos autores foram de 1,63\%, 1,28\% e 1,61\% para as misturas diesel-biodiesel, biodieselbenzeno e biodiesel-tolueno, respectivamente. Segundo os autores, diante dos resultados encontrados, a Equação de Andrade representa satisfatoriamente a variação da viscosidade cinemática com a temperatura em todos os sistemas estudados. Gülüm e Bilgin (2017) também estudaram o modelo da Equação 1, com dados de viscosidade cinemática das misturas BX formados por biodiesel de avelã, nas proporções de B5, B10, B15, B20, B50 e B75, nas temperaturas de $283 \mathrm{~K}, 293 \mathrm{~K}, 303 \mathrm{~K}$ e $313 \mathrm{~K}$. Os autores encontraram erros médio e máximo, e coeficiente de determinação de 2,6532\%, 9,0535\% 
e 0,9412, respectivamente. Diante dos erros encontrados, pode-se concluir que a Equação 1, representou de modo satisfatório os dados experimentais avaliados.

A composição do biodiesel também se destaca nos modelos estudados para predição de viscosidade cinemática, posto que as propriedades fluidodinâmicas do combustível estão diretamente relacionadas com a constituição do mesmo. 0 modelo estudado por Ramírez-Verduzco (2013), Equação 2, leva em consideração fatores relacionados à composição dos ésteres alquílicos de ácidos graxos (EAAG) que compõem o biodiesel. $\mathrm{O}$ autor utilizou dados de viscosidade dinâmica provenientes da literatura. Os biodiesel estudados foram misturas binárias dos biodiesel de coco-canola, algodão-babaçu e soja-babaçu.

$$
\mu_{E A A G}(M, N, T)=\exp \left[a_{1}+a_{2} \cdot \ln (M)+a_{3} \cdot N+a_{4} / T\right]
$$

Sendo $\mu_{\text {EAAG }}$ e M, a viscosidade dinâmica em mPa.s e massa molar dos EAAG em g.mol-1, respectivamente, e $\mathrm{N}$ o número de ligações duplas presentes na cadeia carbônica principal dos EAAG.

Tal como a Equação de Andrade, o modelo apresentado na Equação 2 é exponencial e exibe uma relação inversamente proporcional entre viscosidade cinemática e temperatura. 0 modelo de viscosidade cinemática estudado por Ramírez-Verduzco (2013) considera fatores constitutivos do biodiesel, além do fator temperatura. 0 autor utilizou a Equação de Grunberg-Nissan (Equação 3) aliada à Equação 2 para calcular a viscosidade cinemática das misturas $\mathrm{BX}$, partindo de sua composição de EAAG e desconsiderando o termo associado às interações entre os componentes.

$$
\mu_{b}=\sum_{i=1}^{n}\left[w_{E A A G} \cdot \ln \left(v_{E A A G}\right)\right]
$$

Sendo $\mu_{\mathrm{b}}$ a viscosidade dinâmica do biodiesel em mPa.s, w WAAG e $\mu_{\text {EAAG }}$ a fração mássica e a viscosidade dinâmica dos EAAG's, em mPa.s, que compõem o biodiesel, respectivamente. 0 autor encontrou erros máximos e médios de 17,04 e 6,39\%, respectivamente, o que, segundo o mesmo, indica que o modelo empírico estudado reproduz satisfatoriamente os dados experimentais.

Pham et al. (2018) estudaram um modelo de polinomial que relacionava a viscosidade cinemática com a porcentagem de biodiesel presente na mistura BX através da Equação 4. Os autores avaliaram os biodiesel produzidos pelos óleos de coco, de canola e residual, nas proporções de misturas de B5, B10, B20, B40, B50, B60, B75 e B100. Para o modelo da Equação 4, os autores utilizaram os dados de viscosidade cinemática a $30^{\circ} \mathrm{C}$.

$$
v(X)=a_{1}+a_{2} \cdot X+a_{3} \cdot X^{2}
$$

Onde $v$ é a viscosidade cinemática em cSt e X é a porcentagem de biodiesel presente na mistura BX. 
Diante da comum utilização de misturas BX, visando a redução do uso do diesel puro, modelos que predizem a viscosidade cinemática em função da fração de biodiesel presente na mistura BX, tornam-se relevantes, como observado na Equação 4. 0 modelo avaliado por Pham et al. (2018), tal como os modelos das Equações 1 e 2, evidencia a relação não linear entre a viscosidade cinemática e a porcentagem de biodiesel presente na mistura BX, onde uma relação quadrática foi estudada pelos autores. Para tal modelo, os autores obtiveram erros máximos de 2,8585\%,2,9923\% e 3,3614\% para os biodiesel de coco, canola e óleo residual, respectivamente, quando comparados os dados empíricos com os valores preditos pela Equação 4 . 0 coeficiente de determinação mínimo foi de 0,9943 para o biodiesel de óleo residual. Segundo os autores, esses resultados demonstram que o modelo avaliado apresenta alto nível de confiança na predição da viscosidade cinemática dos BX.

\section{Materiais e métodos}

Dados experimentais de viscosidade cinemática dos biodiesel de algodão (B100-A), soja (B100-S) e sebo bovino (B100-SB) e das misturas B5, B10, B20, B30, B40 e B50, e do diesel de petróleo (B0), obtidos por Cavalcanti (2013), foram analisados, na faixa de temperatura entre 293,15 a 363,15 K, em intervalos de $10 \mathrm{~K}$. Através de regressão não linear, esses dados experimentais foram adequados a um modelo exponencial, proposto com base na literatura e na análise do comportamento desses resultados empíricos. Os parâmetros do modelo foram obtidos através do Matlab, utilizando o aplicativo Curve Fitting, que tem como objetivo gerar parâmetros para o modelo de modo que os valores preditos se aproximem ao máximo dos experimentais.

O coeficiente de determinação $\left(\mathrm{R}^{2}\right)$ foi ajustado para os parâmetros de cada tipo de biodiesel. Os erros percentuais relativos (EPR) foram calculados para os três casos estudados, utilizando a Equação 5.

$$
E P R=\sum_{i=1}^{N}\left|v_{\text {exp }}-v_{\text {mod }} / v_{\text {exp }}\right| \cdot(100 / N)
$$

Sendo, o resíduo $\left(v_{\text {exp }}-v_{\text {mod }}\right)$, calculado pela diferença entre os valores empíricos e modelados, $v_{\exp }$ os valores empíricos e $\mathrm{N}$ o número de pontos experimentais. Foi realizada a análise dos resíduos gerados pelo modelo proposto.

0 teste do qui-quadrado $\left(\mathrm{X}^{2}\right)$ é um método de analisar se um conjunto de dados, dividido em $\mathrm{K}$ classes, adequa-se a uma determinada hipótese, assumindo (K-1) graus de liberdade e estabelecendo o nível de confiança de 95\% para esse trabalho. Através do método do qui-quadrado (Equação 6), foi analisado se o conjunto de dados experimentais de viscosidade cinemática dos biodiesel avaliados e suas misturas BX se adequam ao modelo proposto, que é a hipótese nula, aceitando o nível de confiança supracitado.

$$
X_{\text {calc }}^{2}=\sum_{i=1}^{N}\left[\left(v_{\text {exp }}-v_{\text {mod }}\right)^{2} / v_{\text {mod }}\right]
$$

\section{Resultados e discussão}

Analisando os dados de viscosidade cinemática dos biodiesel de algodão, soja e sebo bovino e misturas BX em função da fração do biodiesel presente nas misturas e da 
temperatura, foi observada não linearidade para as duas variáveis, tal como proposto pelos modelos estudados por Geacai et al. (2015), por Ramírez-Verduzco (2013) e por Pham et al. (2018), Equações 1, 2 e 4, respectivamente. 0 modelo proposto neste trabalho está representado na Equação 7.

$$
v(T, X)=\exp \left(a_{1}+a_{2} \cdot X+a_{3}\left(X^{2}\right)+a_{4} \cdot(X / T)+a_{5} / T+a_{6} / T^{2}\right)
$$

Sendo $v$ a viscosidade cinemática em $\mathrm{cSt}\left(\mathrm{m}^{2} \cdot \mathrm{s}^{-1}\right), \mathrm{X}$ a fração de biodiesel presente na mistura BX, T a temperatura em $\mathrm{K}$ e $\mathrm{a}_{\mathrm{i}}$ os parâmetros do modelo.

Com base na Equação 7, nota-se que foi estudado um modelo exponencial para predição da viscosidade cinemática, como o Modelo de Andrade. Além disso, o polinômio presente na Equação 7 assume que a viscosidade cinemática se relaciona de forma diretamente proporcional com o termo $\mathrm{X}$ e inversamente com a temperatura. Os parâmetros $\mathrm{a}_{\mathrm{i}}$ da Equação 7 estão presentes na Tabela 1.

Tabela 1. Parâmetros $\mathrm{a}_{\mathrm{i}}$ da Equação 7 para mistura BX-A, BX-S e BX-SB.

\begin{tabular}{|l|c|c|c|c|c|c|c|}
\hline Parâmetro & $\mathbf{a}_{\mathbf{1}}$ & $\mathbf{a}_{\mathbf{2}} \cdot \mathbf{1 0}^{\mathbf{2}}$ & $\mathbf{a}_{\mathbf{3}}$ & $\mathbf{a}_{\mathbf{4}}(\mathbf{K})$ & $\mathbf{a}_{\mathbf{5}}(\mathbf{K})$ & $\mathbf{a}_{\mathbf{6}} \cdot \mathbf{1 0}^{-\mathbf{5}}\left(\mathbf{K}^{\mathbf{2}}\right)$ & $\mathbf{R}^{\mathbf{2}}$ \\
\hline BX-A & $-0,1795$ & 8,868 & 0,1046 & 47,89 & $-1124,0$ & 4,807 & 0,9995 \\
\hline BX-S & $-0,4049$ & $-10,220$ & $-0,0612$ & 139,90 & $-950,6$ & 4,487 & 0,9993 \\
\hline BX-SB & $-0,9540$ & 8,462 & 0,0357 & 162,30 & $-687,5$ & 4,197 & 0,9995 \\
\hline
\end{tabular}

Os resultados exibidos na Tabela 1 foram obtidos através de regressões não lineares dos dados empíricos de viscosidade cinemática de cada tipo de biodiesel estudado, de modo a ajustar o modelo da Equação 7 à esses dados. $0 \mathrm{R}^{2}$ mínimo é 0,9993, referente a mistura BX-S.

Aplicando os valores dos parâmetros $\mathrm{a}_{\mathrm{i}}$, exibidos na Tabela 1, na Equação 7, obtémse equações que correspondem às superfícies modeladas de viscosidade cinemática de cada tipo de biodiesel. As Figuras de 1 a 3 representam os dados experimentais de Cavalcanti (2013) e as superfícies modeladas para as misturas BX-A, BX-S e BX-SB a partir do modelo proposto no presente trabalho.

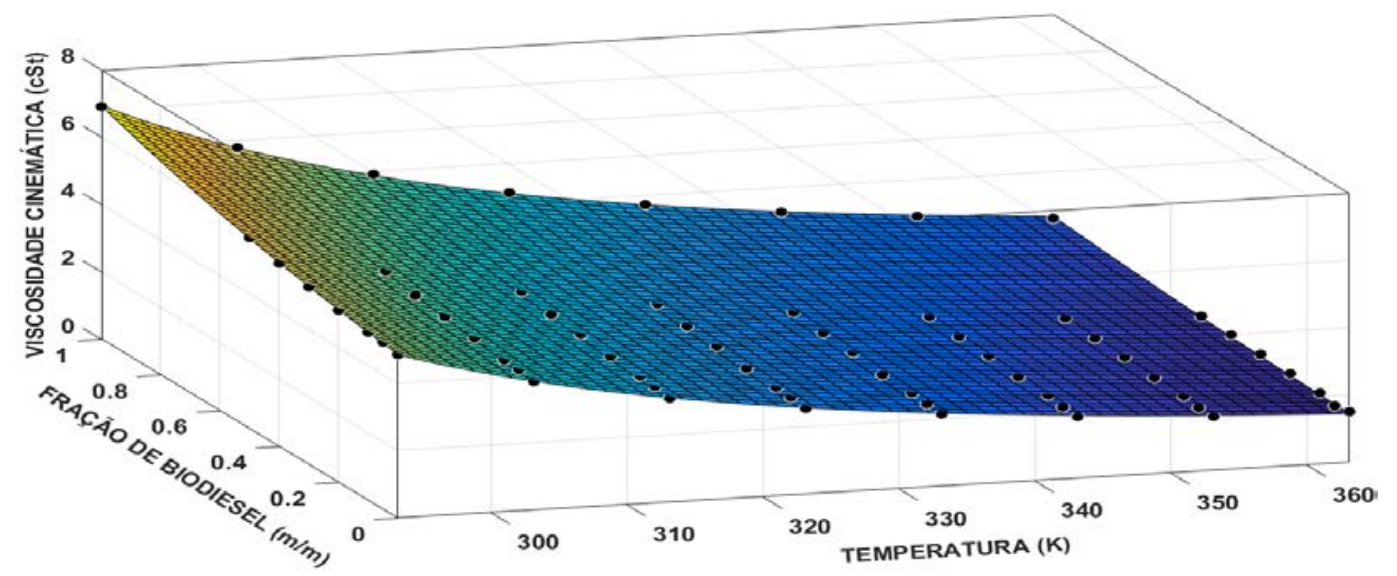

Figura 1. Superfície modelada de viscosidade cinemática para a mistura BX-A. 


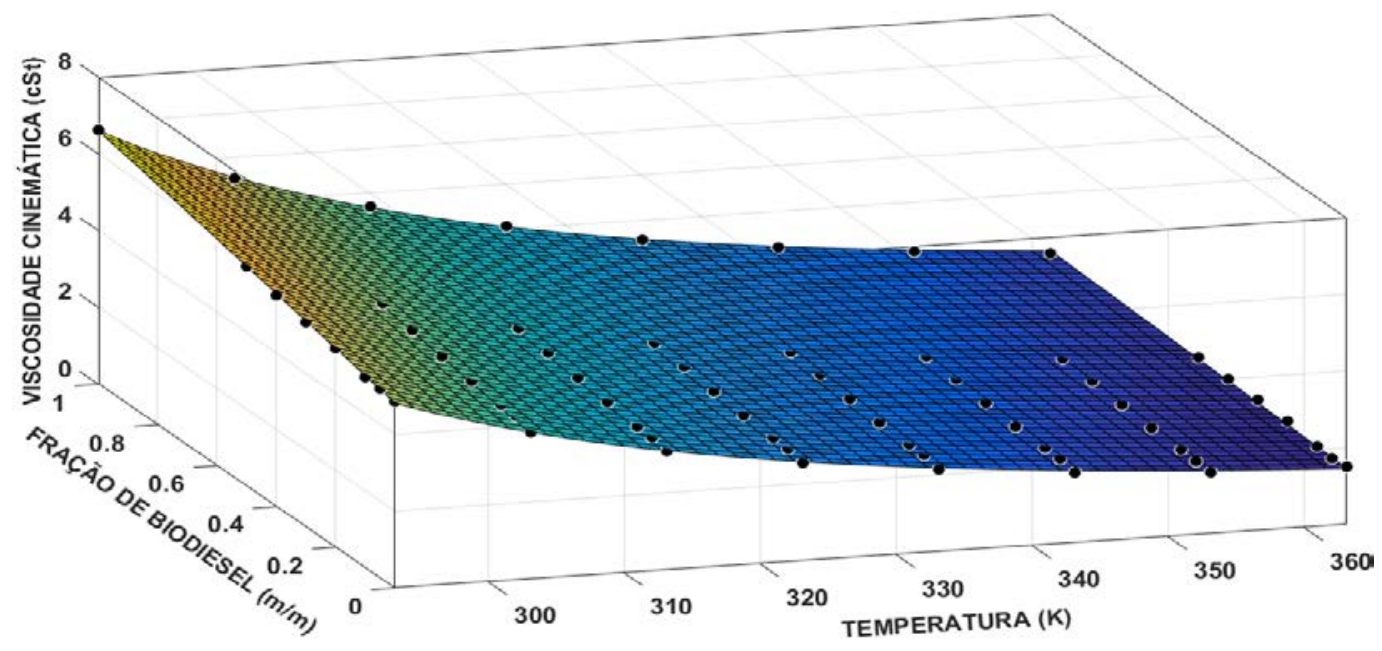

Figura 2. Superfície modelada de viscosidade cinemática para a mistura BX-S.

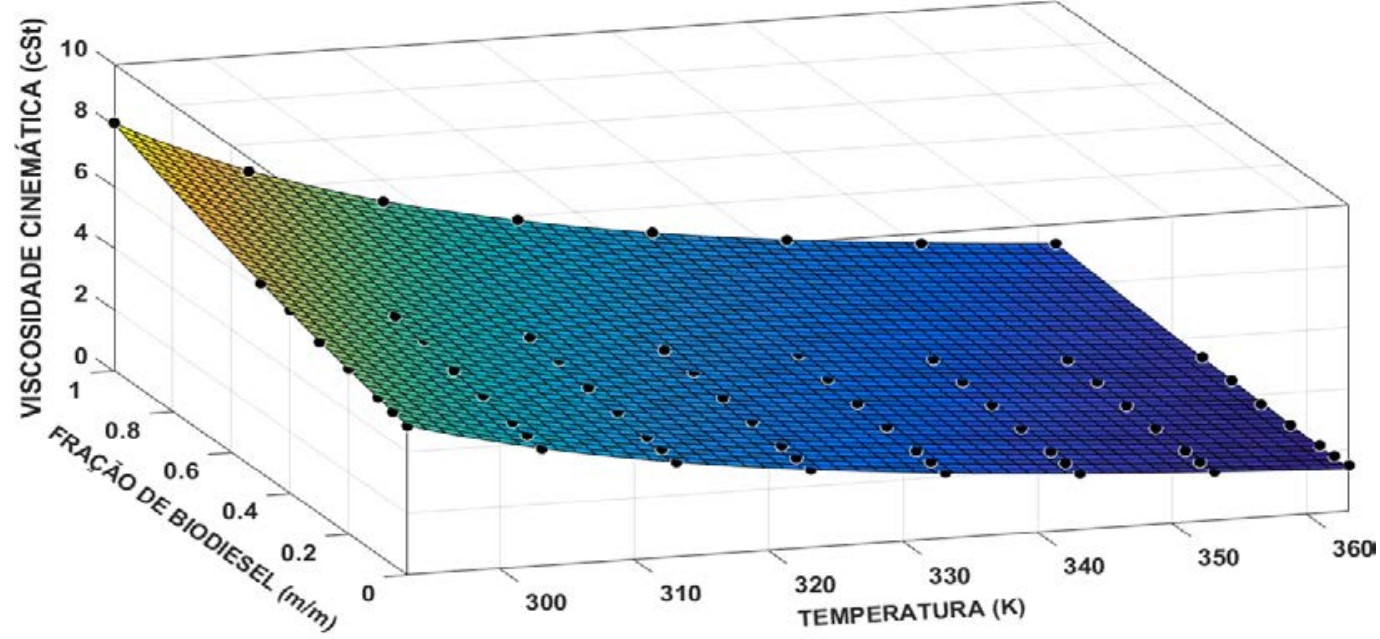

Figura 3. Superfície modelada de viscosidade cinemática para a mistura BX-SB.

A partir das Figuras de 1 a 3, nota-se que os pontos experimentais estão contidos nas superfícies, evidenciando que as superfícies modeladas aproximam-se satisfatoriamente aos dados empíricos, constatação ratificada com a análise dos valores de EPR, presentes na Tabela 2.

Tabela 2. Valores de EPR gerados pela Equação 7 para as misturas BX-A, BX-S e BX-SB.

\begin{tabular}{|l|c|}
\hline Biodiesel & EPR (\%) \\
\hline BX-A & 1,0976 \\
\hline BX-S & 1,2338 \\
\hline BX-SB & 0,9663 \\
\hline
\end{tabular}


Avaliando a Tabela 2, nota-se que o maior valor de EPR é 1,2338\%, correspondendo a mistura BX-S. Esse desvio é aceitável, posto que encontra-se próximo aos valores informados pela literatura de trabalhos semelhantes, como 1,63\% obtido por Geacai et al. (2015), 6,39\% por Ramírez-Verduzco (2013) e 3,3614\% por Pham et al. (2018).

A diferença entre os valores empíricos e aqueles obtidos via modelo são os resíduos. A análise de resíduos permite avaliar se o modelo estudado apresenta tendenciosidade. As Figuras de 4 a 6 apresentam as análises de resíduos para as misturas BX-A, BX-S e BX-SB, gerados pela Equação 7.

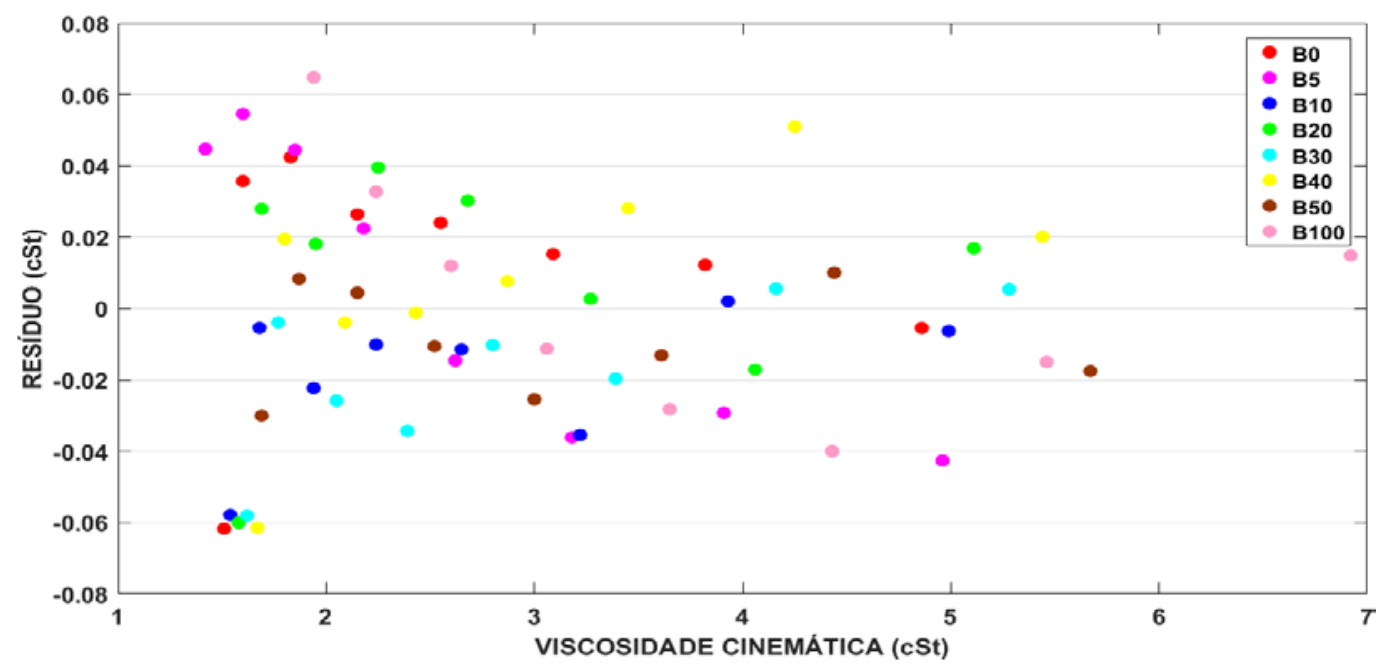

Figura 4. Análise de resíduo gerado pela Equação 7 para mistura BX-A.

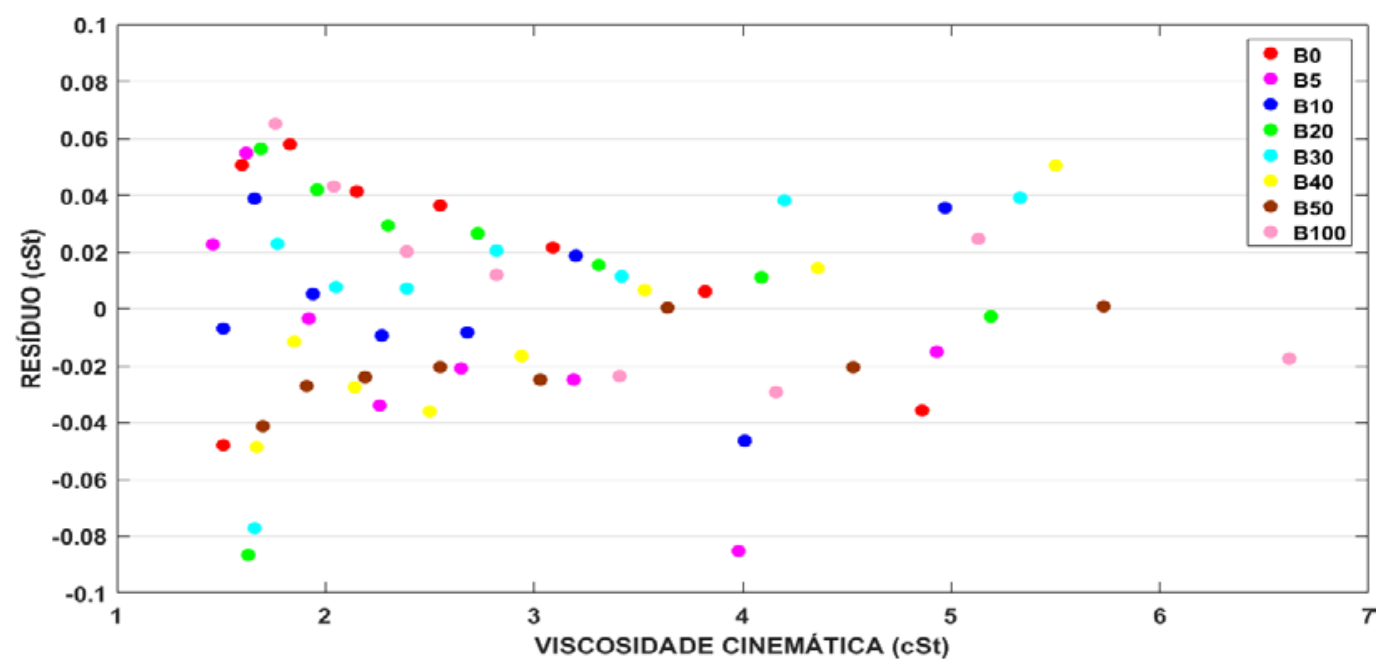

Figura 5. Análise de resíduo gerado pela Equação 7 para mistura BX-S. 


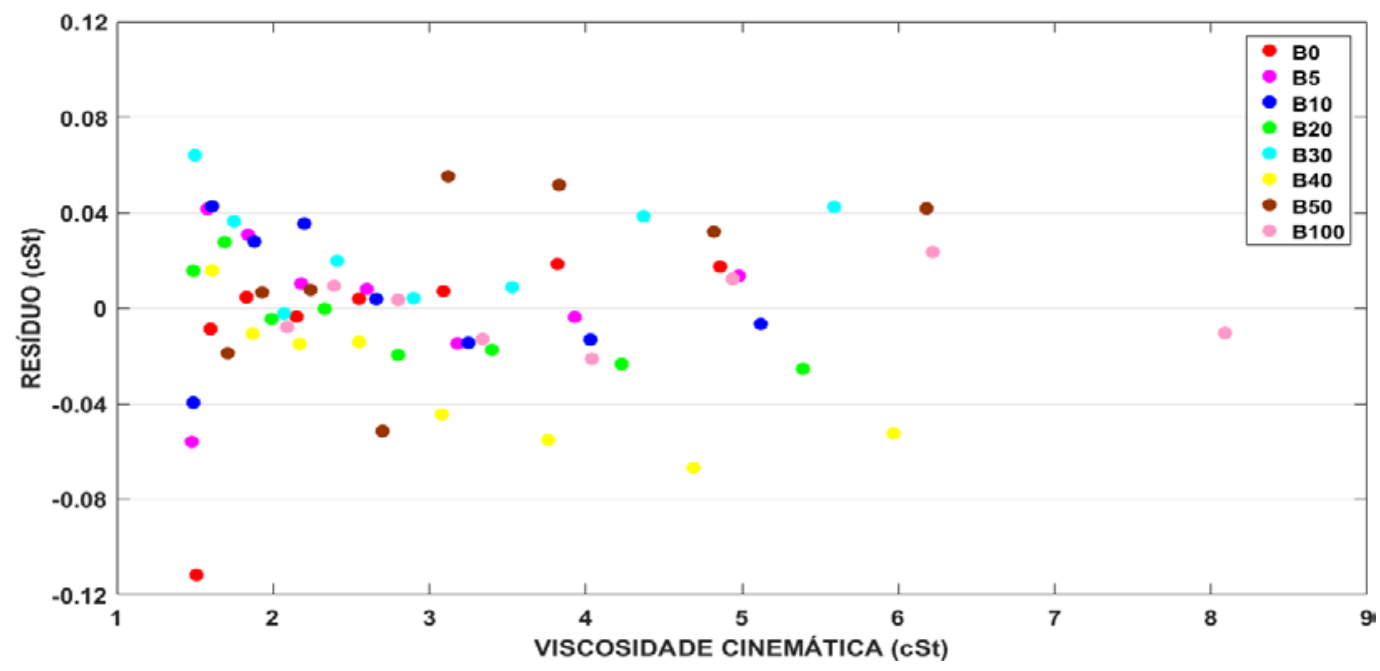

Figura 6. Análise de resíduo gerado pela Equação 7 para mistura BX-SB.

Baseando-se nas Figuras de 4 a 6, percebe-se que o modelo da Equação 7 não se mostra tendencioso em nenhum caso estudado, exibindo uma distribuição aleatória dos pontos em torno da origem.

Uma avaliação mais robusta quanto validade do modelo em representar os dados experimentais pode ser avaliada através do teste qui-quadrado (teste de aderência de modelos). Os valores de qui-quadrado obtidos para as misturas BX-A, BX-S e BX-SB estão apresentados na Tabela 3.

Tabela 3. Dados de $\mathrm{X}^{2}$ calculado para os BX-A, BX-S e BX-SB.

\begin{tabular}{|l|c|}
\hline Biodiesel & $\mathbf{X}^{2}$ calculado \\
\hline BX-A & 0,0287 \\
\hline BX-S & 0,0358 \\
\hline BX-SB & 0,0287 \\
\hline
\end{tabular}

Os dados foram divididos em 8 classes, logo o grau de liberdade é 7. Com um nível de confiança de 95\%, o valor de $\mathrm{X}^{2}$ tabelado é de 14,067. Portanto, observando a Tabela 3, nota-se que em todos os casos o $\mathrm{X}^{2}$ calculado $<\mathrm{X}^{2}$ tabelado. Diante disso, o modelo da Equação 7 adequa-se satisfatoriamente aos dados experimentais de viscosidade cinemática para as misturas BX-A, BX-S e BX-SB, no intervalo de temperatura avaliado.

\section{Conclusão}

O modelo proposto para viscosidade cinemática mostrou-se adequado em representar os dados experimentais dos biodiesel de algodão, soja e sebo bovino e suas misturas BX, no intervalo de temperatura estudado. Os parâmetros do modelo foram obtidos com $\mathrm{R}^{2}$ mínimo de 0,993 . Os valores de EPR calculados são aceitáveis e abaixo dos relatados em trabalhos similares. Foi observada aleatoriedade dos resíduos gerados pelo modelo em torno da origem para todos os casos estudados, através da análise de resíduos. 0 teste do qui-quadrado indicou que o modelo proposto é estatisticamente adequado para 
os três biodiesel estudados, posto que em todos os casos $\mathrm{X}^{2}$ calculado $<\mathrm{X}^{2}$ tabelado. Portanto, o modelo proposto neste trabalho mostrou apto em predizer a viscosidade cinemática dos biodiesel de algodão, soja e sebo bovino, na faixa de temperatura estudada, permitindo seu uso em outras frentes de estudo.

\section{Conflito de interesse}

Os autores declaram não haver conflito de interesses.

\section{Referências}

Aghel, B.; Mohadesi, M.; Ansari, A.; Maleki, M. Pilot-scale production of biodiesel from waste cooking oil using kettle limescale as a heterogeneous catalyst. Renewable Fuel, v. 142, p. 207-214, 2019. https://doi.org/10.1016/j.renene.2019.04.100

Cavalcanti, L. A. P. Reologia e melhoramento das propriedades de escoamento a frio de biodiesel e suas misturas BX. Recife: Universidade Federal de Pernambuco, 2013. (Tese de doutorado).

Cavalcanti, L. A. P. Modelos de regressão aplicados para predição das propriedades físicas das misturas do biodiesel de sebo bovino com o diesel. CIATEC - UPF, v. 8, n. 2, p. 22-33, 2016. https://doi.org/10.5335/ciatec.v8i2.6115

Geacai, S.; Iulian, O.; Nita, I. Measurement, correlation and prediction of biodiesel blends viscosity. Fuel, v. 143, p. 268-274, 2015. https://doi.org/10.1016/j.fuel.2014.11.041

Gülüm, M.; Bilgin, A. Measurements and empirical correlations in predicting biodieseldiesel blends' viscosity and density. Fuel, v. 199, p.567-577, 2017. https://doi.org/10.1016/j.fuel.2017.03.001

Kanaveli, I.-P.; Atzemi, M.; Lois, E. Predicting the viscosity of diesel/biodiesel blends. Fuel, v. 199, p. 248-263, 2017. https://doi.org/10.1016/j.fuel.2017.02.077

Pham, M. T.; Hoang, A. T.; Le, A. T.; Tawaha, A. R. M. S. A.; Dong, V. H.; Le, V. V. Measurements and prediction of the density and viscosity of biodiesel blends. International Journal of Technology, $\quad$ v. 5, $\quad$ n. 5, p. 1015-1026, 2018. https://doi.org/10.14716/ijtech.v9i5.1950

Ramirez-Verduzco, L. F. R. Density and viscosity of biodiesel as a function of temperature: Empirical models. Renewable and Sustainable Energy Reviews, v. 19, p. 652-665, 2013. https://doi.org/10.1016/j.rser.2012.11.022

Wang, S.; Sui, M.; Luo, H.; Li, F.; Zhai, Y. The study on the influence of oxidation degree and temperature on the viscosity of biodiesel. Green Processing and Synthesis, v. 9, n. 1, p. 182-190, 2020. https://doi.org/10.1515/gps-2020-0019 